\title{
Immune subtyping for pancreatic cancer with implication in clinical outcomes and improving immunotherapy
}

Jingkai Liu, Qiaofei Liu, Xiang Zhang, Ming Cui, Tong Li, Yalu Zhang and Quan Liao* (1)

\begin{abstract}
Background: Emerging evidence has shown that intra-tumor immune features are associated with response to immune checkpoint blockade (ICB) therapy. Accordingly, patient stratification is needed for identifying target patients and designing strategies to improve the efficacy of ICB therapy. We aimed to depict the specific immune features of patients with pancreatic cancer and explore the implication of immune diversity in prognostic prediction and individualized immunotherapy.
\end{abstract}

Methods: From transcriptional profiles of 383 tumor samples in TCGA, ICGC, and GEO database, robust immune subtypes which had different response immunotherapy, including ICB therapy, were identified by consensus clustering with five gene modules. DEGs analysis and tumor microarray were used to screen and demonstrate potential targets for improving ICB therapy.

Results: Three subtypes of pancreatic cancer, namely cluster 1-3 (C1-C3), characterized with distinct immune features and prognosis, were generated. Of that, subtype $\mathrm{C} 1$ was an immune-cold type in lack of immune regulators, subtype C2, with an immunosuppression-dominated phenotype characterized by robust TGF $\beta$ signaling and stromal reaction, showed the worst prognosis, subtype C3 was an immune-hot type, with massive immune cell infiltration and in abundance of immune regulators. The disparity of immune features uncovered the discrepant applicability of anti-PD-1/PD-L1 therapy and potential sensitivity to other alternative immunotherapy for each subtype. Patients in C3 were more suitable for anti-PD-1/PD-L1 therapy, while patients in the other two clusters may need combined strategies targeted on other immune checkpoints or oncogenic pathways. A promising target for improving anti-PD-1/ PD-L1 treatment, TGM2, was screened out and its role in the regulation of PD-L1 was investigated for the first time.

Conclusion: Collectively, immune features of pancreatic cancer contribute to distinct immunosuppressive mechanisms that are responsible for individualized immunotherapy. Despite pancreatic cancer being considered as a poor immunogenic cancer type, the derived immune subtypes may have implications in tailored designing of immunotherapy for the patients. TGM2 has potential synergistic roles with ICB therapy.

Keywords: Pancreatic cancer, Heterogeneity, Immune cell, Immune checkpoints, Immunotherapy, Transglutaminase 2

*Correspondence: lqpumc@126.com

Department of General Surgery, Peking Union Medical College Hospital, Peking Union Medical College, Chinese Academy of Medical Sciences, Beijing 100730, China

\begin{abstract}
Introduction
Pancreatic ductal adenocarcinoma (PDAC) has been striking a heavy burden on human health by increasing worldwide incidence and less than $9 \%$ survival rate [1]. Advances in chemotherapy regimens over the last two decades have only modestly prolonged the overall
\end{abstract}


survival of the patients [2]. More effective treatments are still needed for PDAC patients. A promising ICB therapy, programmed cell death protein 1 (PD-1)/programmed death 1 ligand 1(PD-L1) antibodies, has yielded significant clinical efficacy in some tumor types [3]. However, low response rate and limited patients benefited from single-agent ICB were observed in PDAC, which can be attributed to the low immunogenicity and diverse immunosuppression mechanisms [4]. To overcome the drug resistance, combination with other ICB targets or other therapeutic modalities has been regarded as a hopeful solution [5]. While the precondition for making an appropriate combination treatment strategy is a reasonable method for patient stratification based on similar characteristics of the immune response.

Over the last decade, substantial progression in molecular subtyping for PDAC has facilitated the understanding of molecular pathogenesis and provided clues for advanced therapy designing [6]. However, to date, there is still lacking an immune feature-based molecular subtyping for better understanding the heterogeneity of immune response and reasons for the inefficiency of ICB therapy.

A recent pan-cancer study revealed a widely suitable immune-subtyping method based on five immune signatures which provided a potential roadmap for PDAC [7]. Herein three distinct immune subtypes of PDAC were presented based on these five immune signature modules. Each subtype showed distinct immune cell composition and expression patterns of immunomodulators, which provided a reasonable explanation for their survival discrepancy and inefficacy of single-agent ICB therapy. Furthermore, we screened out a potential immunosuppression-related gene, transglutaminase 2 (TGM2). TGM2 is a multifunctional enzyme that, in addition to catalyzing protein crosslinking, can also serve in the regulation of signaling pathways by constitutively activating the key modulators, such as p53, Akt, and NF- $k B$ [8-13]. The aberrant expression of TGM2 has been demonstrated to be linked with a series of aggressive phenotypes of tumor cells, such as tumor growth, metastasis, epithelial-mesenchymal transition, and cancer stem cell property $[12,14]$. In vivo, based on orthotopic xenograft mouse models, downregulating the expression of TGM2 by siRNA or shRNA could restrain the tumor growth and improve the treatment effect of Gemcitabine in PDAC $[9,15,16]$. At present, whether or how TGM2 is involved in the immune regulation of PDAC remains unknown. Therefore, we explored the association of TGM2 with the immune microenvironment and investigated its potentially synergistic roles with ICB therapy.

Taken together, these findings provide a conceptional framework to understand the immune response diversity in the tumor microenvironment of PDAC, implicate PDAC patient stratification, and design combination therapeutic strategies based on ICB therapy.

\section{Materials and methods \\ Transcriptional data resources and preprocessing}

Human pancreatic cancer transcriptional profiles were downloaded from public databases, including The Cancer Genome Atlas (TCGA, https://portal.gdc.cancer.gov/), International Cancer Genome Consortium (ICGC, https ://icgc.org/) and Gene Expression Omnibus (GEO, https ://www.ncbi.nlm.nih.gov/geo/). Read count data from TCGA (PAAD) and ICGC (PACA-AU) were normalized in the TMM method by edgeR ( $\mathrm{R}$ package). Then TPM value was calculated respectively and the batch effect was eliminated by Combat. There were 182 TCGA data samples, of which 4 para-cancer samples and 1 nonprimary sample were excluded, and finally, a total of 177 tumor samples were collected. GSE28735 and GSE62452, which are two datasets downloaded from the GEO database with the entire clinical following information, were integrated. The batch effect was eliminated by Combat. The combined datasets of GSE28735 and GSE62452 contained a total of 220 samples, including 114 tumor samples and 106 para-cancer samples. Together with 92 tumor samples from ICGC and 177 tumor samples from TCGA, 383 tumor samples were taken into analysis. To accomplish a combined analysis of tumor statistics, RNASeq and gene array transcriptional data were normalized in the manner of $\mathrm{z}$-score. The flow chart of data processing was shown in Fig. 1.

\section{Patient cohort}

The use of clinical samples and clinical information for this study was approved by the institutional review board of Peking Union Medical Hospital (NO. JS-987). Totally, 97 pairs of tumor and para-tumor normal samples of patients diagnosed with PDAC were collected as the description of our previous study [17]. All of the cases were pathologically confirmed to be PDAC, and R0 radical resection was achieved and at least three cycles of adjuvant chemotherapy were performed for each patient. Histological grading was made according to the 8th edition of the TNM system established by the American Joint Committee on Cancer (AJCC) [18]. None of the patients undergone neoadjuvant chemotherapy.

\section{Immunohistochemistry (IHC) and digital analysis}

Immunohistochemistry staining was implemented in accordance with the previous study [17]. The microarray chip was stained with anti-TGM2 (15100-1-AP, Proteintech, 1:200). Quant Center identified and analyzed the areas of strong positive, moderately positive, weak 


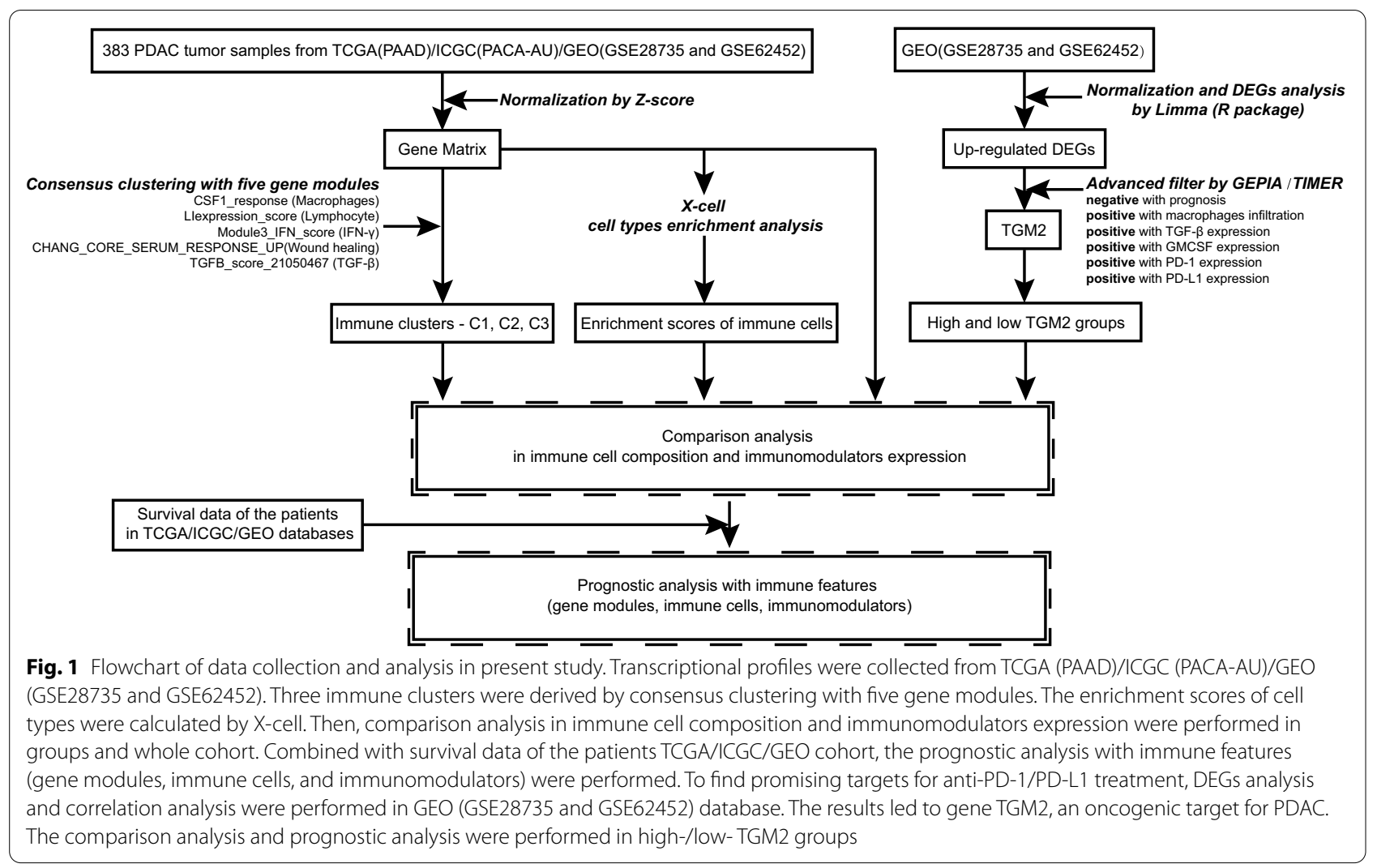

positive, and negative pixels, as well as the percentage of positive pixels, and finally conducted an $\mathrm{H}$-score.

\section{Cell culture}

Human pancreatic cancer cell lines, PANC-1 and Mia $\mathrm{PaCa}-2$, were maintained in DMEM medium supplemented with $10 \%$ fetal bovine serum and $1 \%$ penicillin and streptomycin and cultured in the incubator with $5 \%$ $\mathrm{CO}_{2}$ at $37^{\circ} \mathrm{C}$.

\section{Western blotting}

Total protein was extracted using 2\% SDS lysis buffer including protein phosphatase inhibitor (Applygen, Beijing) and heated for $10 \mathrm{~min}$. Protein samples were separated by $8 \%(\mathrm{v} / \mathrm{v})$ SDS-PAGE gels and transferred onto nitrocellulose membranes (Millipore, Ireland). After blocking in 5\% (w/v) Albumin Bovine-V (BSA-V, Solarbio, China) for $1 \mathrm{~h}$ at room temperature, the protein bands were incubated with primary antibodies at a dilution ratio of $1: 1000$ overnight at $4{ }^{\circ} \mathrm{C}$. The primary antibodies contain anti-GAPDH (FL-335; Santa Cruz Bio technology), anti-TGM2 (Proteintech), anti-PD-L1 (Proteintech), anti-STAT3 (CST) and anti-Phospho-STAT3 (Ser705, CST), anti-Akt (CST), and anti-Phospho-Akt (Ser473, CST), anti-P65 (Abcam) and anti-Phospho-P65 (Ser536, Abcam). The protein bands were incubated in
HRP-conjugated secondary antibodies (Zsbio, China, 1:5000) at room temperature for $1 \mathrm{~h}$.

\section{Transfection assay}

Lentiviral particles for the TGM2 knockdown assay were purchased from Syngen Tech (Beijing, China). Lentiviral particles were added into PANC-1 cells and Mia PaCa-2 cells supplemented with $5 \mu \mathrm{g} / \mathrm{ml}$ polybrene. After $48 \mathrm{~h}$ of transfection, target cells were selected with $1 \mu \mathrm{g} / \mathrm{ml}$ of puromycin for two weeks. The TGM2 knockdown in cells was validated by Western blotting. The sequence of shTGM2 is acquired from a previous study and showed as below: 5'-AAGGGCGAACCACCTGAACAA-3' [15].

\section{Statistical analysis Identification of immune subtypes}

Five immune signature sets, including CSF1_response (Macrophages), LIexpression_score (Lymphocyte), Module3_IFN_score (IFN- $\gamma$ ), TGFB_score_21050467 (TGF- $\beta$ ) and CHANG_CORE_SERUM_RESPONSE_UP (Wound healing) modules, were selected to run clustering analysis. They respectively represented the activation of macrophage/monocytes, overall lymphocyte infiltration, TGF- $\beta$ response, IFN- $\gamma$ response, and wound healing activity in tumor immune microenvironment. GSVA enrichment analysis was conducted and ssGSEA values 
were calculated. Unsupervised clustering with ssGSEA values of samples was conducted by McLust $R$ package, and $\mathrm{K}$ values corresponding to maximized Bayesian Information criterion (BIC) were selected to obtain immune subtypes.

\section{Depiction of molecular and cellular signatures in immune subtypes}

To further understand the cellular and molecular characteristics of the immune microenvironment, we assessed the enrichment degree of 75 immunomodulatory genes and 64 immune cell types. The enrichment of immune cell types and immune-related genes were analyzed by xCel software and ImmuneCellAI (http://bioinfo.life. hust.edu.cn/ImmuCellAI) and R software. Kruskal-wallis test or Wilcox test were used to analyze the differences in enrichment scores of immune cells or immunomodulatory genes among different groups. Boxplot was drawn for the distribution of immune cells. The accuracy of immune cells in predicting survival was analyzed by C-index analysis.

\section{Prognostic analysis}

To evaluate the impact of immune signatures on patients' survival, we performed Kaplan-Meier analysis, univariate and multivariate COX analysis. The results were presented as the mean \pm standard deviation. $C$-index was used to analyze the accuracy of the gene sets model in predicting survival. $p<0.05$ was considered statistically significant.

\section{Identification of immune related gene target}

Based on the gene expression profiles of GSE28735 and GSE62452, differentially expressed genes (DEGs) were collected by limma ( $\mathrm{R}$ package) with filtration of $p$-value $<0.05$ and Fold Change $(\mathrm{FC})>1$. To identify target genes among the DEGs, further filtration was set as negative relation with prognosis, positive relation with the infiltration of macrophages and the expression of PD-L1, PD-1, TGF- $\beta 1$ and GM-CSF and analyzed by the online tool of GEPIA (http://gepia.cancer-pku.cn/) and TIMER (https://cistrome.shinyapps.io/timer/). Set median expression value of target gene as cutoff and we divided the whole samples into high and low expression groups. The distribution of high and low groups in three immune subtypes then was described in a percentage and count manner. The prognostic analysis of the target gene was conducted by survival $R$ package. The influence of immune modulator genes on patients' survival in the above two high and low expression groups was also analyzed.

\section{Results}

Identification of immune subtypes in PDAC

PDAC harbored a highly heterogeneous tumor microenvironment. By performing clustering analysis with five immune signature gene sets, we classified three immune subtypes, namely $\mathrm{C} 1-\mathrm{C} 3$, in the 383 pancreatic tumor samples. The five immune signatures represented the activation of macrophage/monocytes (Macrophages), overall lymphocyte infiltration (Lymphocyte), TGF- $\beta$ response (TGF- $\beta$ ), IFN- $\gamma$ response (IFN- $\gamma$ ), and wound healing activity (Wound healing), respectively.

Five immune signatures showed disparate enrichment patterns in three immune subtypes (Fig. 2a). Subtype C3 had the highest Lymphocyte and Macrophages as well as a favorable enrichment in IFN- $\gamma$, indicating an immune-hot phenotype (Fig. 2b). Subtype $\mathrm{C} 1$ had generally poor enrichment scores, especially the lowest enrichment in IFN- $\gamma$, TGF- $\beta$, and Wound healing, indicating an immune-cold phenotype (Fig. 2b). In comparison, subtype $\mathrm{C} 2$ had the highest TGF- $\beta$ enrichment scores and the lowest Lymphocytes enrichment scores, indicating an immune-suppressive phenotype (Fig. 2b). Meanwhile, C2 also had the highest Wound healing enrichment scores indicating an active tissue remolding (Fig. 2b).

\section{Cellular composition and immunomodulators diversity of immune subtypes}

Immune cell composition varied across three subtypes. Subtype C3, the immune-hot phenotype, had dominant cellular enrichment scores with the highest $\mathrm{T}$ lymphocytes (both $\mathrm{CD}^{+}$and $\mathrm{CD} 4^{+}$), NKT cells, dendritic cells (DCs), macrophages, and B cells as well as granulocytes (Fig. 3a, b). Meanwhile, three subtypes displayed distinct expression patterns of immunomodulators (Fig. 3c). Compared with subtype $\mathrm{C} 1$ and $\mathrm{C} 2$, subtype $\mathrm{C} 3$ had higher CD27 and inducible synergistic co-stimulation molecules (ICOS) expression, indicating a favorable lymphocyte activation. In addition, higher IL2, IFN- $\gamma$, and TNF families (e.g. CD40) represents a favorable cellular immunity and anti-tumor ability of C3 (Fig. 3d). The expression pattern of inhibitory immune regulators varied from three subtypes. In addition to a higher TGF- $\beta 1$, subtype $\mathrm{C} 3$ had higher immune checkpoint expression of CD274 (PD-L1, also as B7-H1), PDCD1 (PD-1), CTLA4, TIGIT, LAG3, HAVCR2 (TIM3) but lower expression of CD276 (B7-H3), and VTCN1 (B7-H4), compared with $\mathrm{C} 1$ and $\mathrm{C} 2$ (Fig. 3d, e).

Opposite enrichment patterns of immune-infiltrating cells were found between subtype $\mathrm{C} 1$ and C2. Subtype $\mathrm{C} 1$ had higher enrichment scores in T cells, NKT cells, DCs, and B cells but lower in macrophages, while C2 had higher scores in macrophages but lower scores in $\mathrm{T}$ 

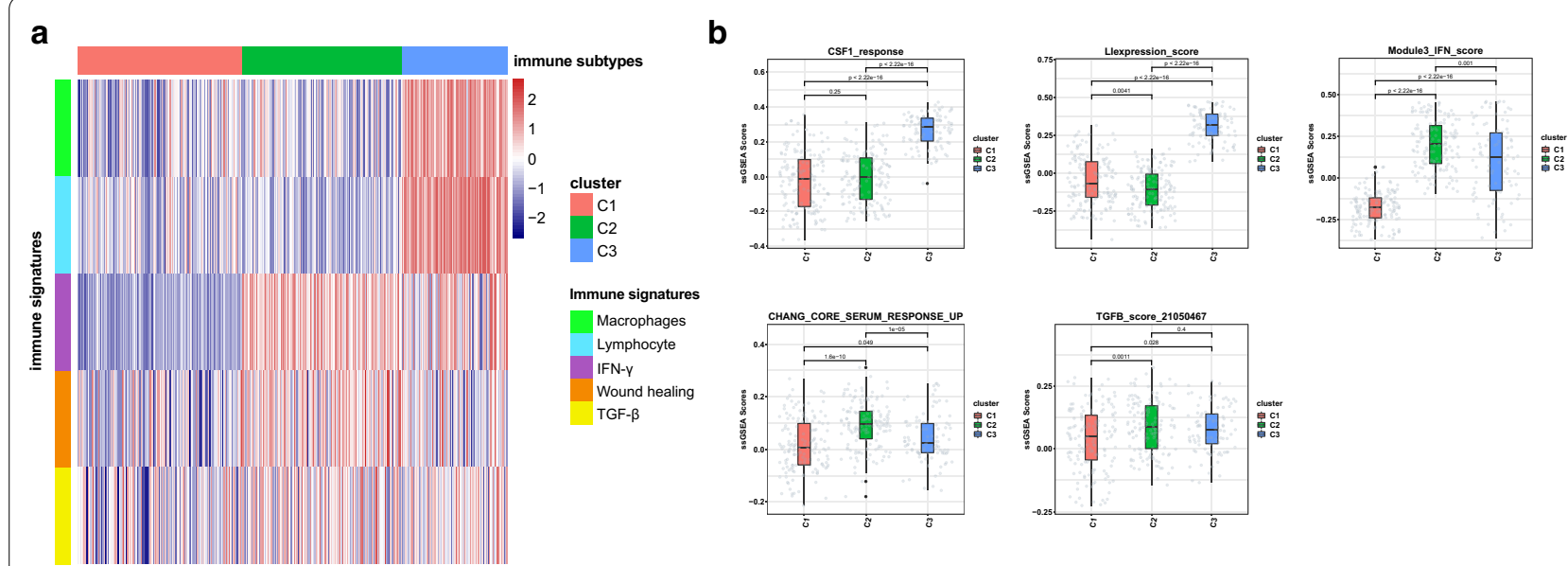

Fig. 2 Immune subtypes of PDAC and immune signatures in TCGA and ICGC and GEO mix. a Immune subtypes of PDAC: column was for tumor samples and row was for five immune signatures. $\mathbf{b}$ Enrichment comparison of the five immune gene modules across three immune subtypes. Gene modules include CSF1_response (correspond to Macrophages), Llexpression_score (correspond to Lymphocyte), Module3_IFN_score (correspond to IFN- $\gamma$ ), CHANG_CORE_SERUM_RESPONSE_UP (correspond to Wound healing), and TGFB_score_21050467 (correspond to TGF- $\beta$ )

cells, NKT cells, DCs, and B cells (Fig. 3a, b), indicating a stronger immunosuppressive microenvironment of subtype $\mathrm{C} 2$. On the other hand, both $\mathrm{C} 1$ and $\mathrm{C} 2$ showed a poor expression of stimulatory immunomodulators. As for the expression discrepancy of immune checkpoints, subtype C1 was mainly enriched in VTCN1, while C2 was dominated by CD274, CD276, and VTCN1 (Fig. 3d, e), which may have the clinical implication for further designing tailored immunotherapy strategy.

The stromal and immune scores of tumor samples were calculated by ESTIMATE software. Different from the discrete distribution of $\mathrm{C} 1$ and $\mathrm{C} 2$, subtype $\mathrm{C} 3$ was mostly enriched in the zones with high immune scores, which indicated a robust immune activity in C3 (Fig. 3f).

\section{Prognosis analysis of immune subtypes}

The association between immune subtypes and patients' survival was also analyzed. The immune-suppressive subtype $\mathrm{C} 2$ had the worst prognosis, while $\mathrm{C} 1$ and $\mathrm{C} 3$ had relatively favorable prognosis $(\mathrm{C} 1: \mathrm{C} 2: \mathrm{C} 3, p=0.0019$, Fig. $4 \mathrm{a} ; \mathrm{C} 1: \mathrm{C} 2, p=0.00094 ; \mathrm{C} 1: \mathrm{C} 3, \mathrm{p}=0.24 ; \mathrm{C} 2: \mathrm{C} 3$, $p=0.038$, Additional file 1: Figure S1). The results of $\mathrm{K}-\mathrm{M}$ analysis revealed that IFN- $\gamma(p=0.001)$ and Wound healing $(p=5.645 \mathrm{e}-04)$ modules were inversely correlated with patients' overall survival (Fig. 4b). The univariate and multivariate cox analyses also showed the same results (Fig. 4c and Table 1). Furthermore, with concordance index (CI) analysis, we evaluated the validity of the five immune signatures in survival prediction. The results showed that IFN- $\gamma$, Wound healing and TGF- $\beta$ modules had favorable prediction accuracy on patient survival among the three subtypes (Fig. 4d). Especially, Wound healing module in C2, Macrophages module in C3, TGF- $\beta$ and Lymphocyte module in $C 1$ seemed to be the best predictor for each subtype, respectively (Fig. $4 \mathrm{~d}$ ).

The prognostic analysis showed that NKT cells correlated with a favorable prognosis $(p=0.014)$ (Fig. 5a) while macrophages were associated with a poor prognosis $(p=0.0031)$ (Fig. 5b). The CI analysis showed that both NKT cells and macrophages could serve as favorable predictors for patients' survival in C2 (Fig. 5c). Although other cell types showed no significant correlation with patients' survival in our study (Additional file 1: Figure S2), immune cells especially $\mathrm{CD}^{+} \mathrm{T}$ cells, NKT cells, and $\mathrm{B}$ cells showed a greater impact on patients' survival based on CI scores (Fig. 5d).

\section{TGM2 was involved in the immune-suppressive} microenvironment in PDAC and high TGM2 expression predicted poorer survival

To find the underlying target for anti-PD-1/PD-L1 treatment, we performed DEGs analysis with the two GEO datasets (GSE62452 and GSE28735). Among all the upregulated DEGs, following the set filters, the result led to the gene TGM2 (Additional file 1: Figure S3). With IHC analysis in our tissue microarray, we also verified that TGM2 was elevated in tumor tissue compared with adjacent normal tissue (Fig. 6a) and had a negative impact on prognosis (Fig. 6b). In addition, survival analysis within the TCGA-ICGC-GEO cohort verified the prognostic impact of TGM2 (Additional file 1: Figure S4).

Besides, the expression patterns of TGM2 across the three subtypes were different. Most patients comprising subtype C2 had high TGM2 expression, while subtype 

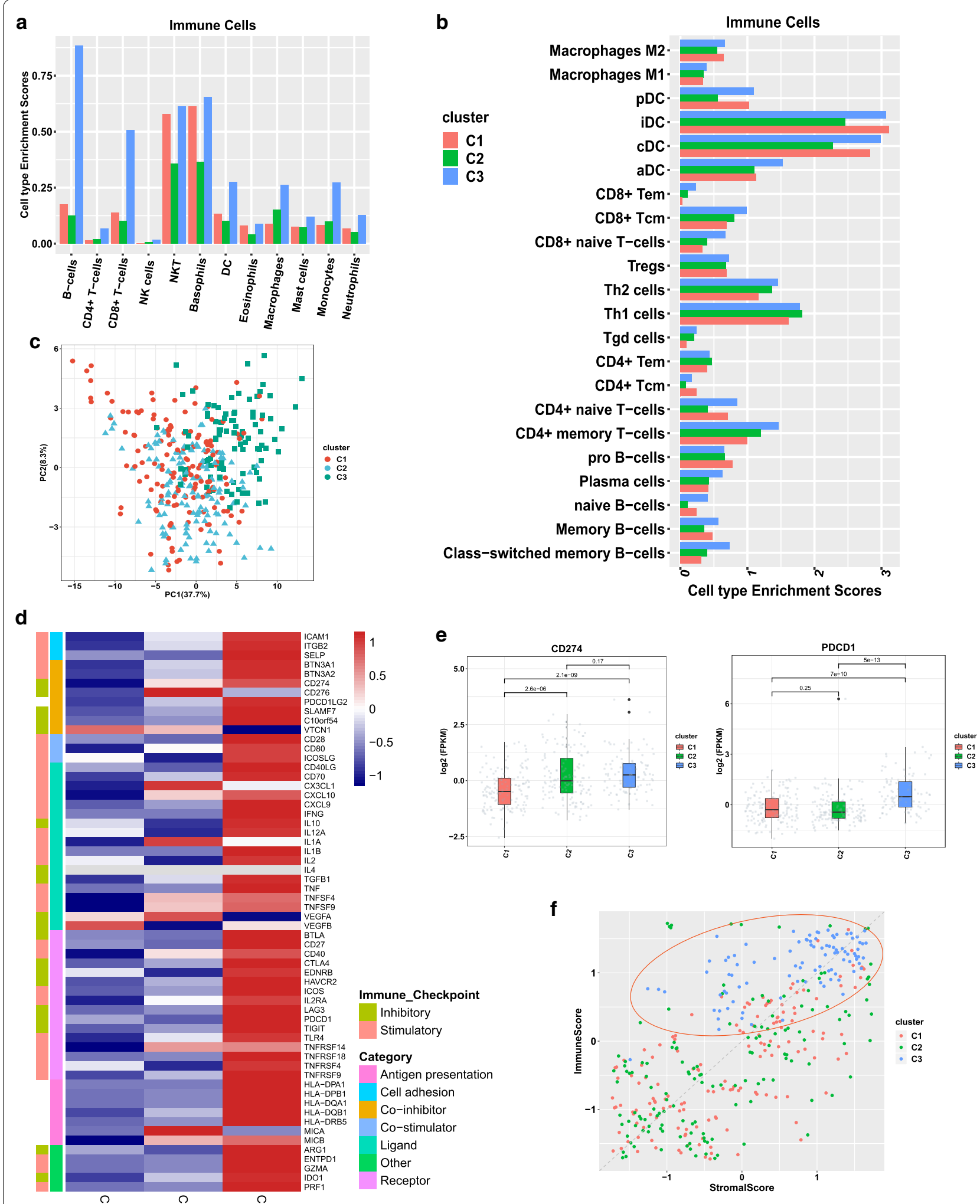

Fig. 3 Immune cell composition and expression patterns of IMs in three subtypes. a, b Immune cell composition of three immune subtypes. c PCA analysis of IMs genes. $\mathbf{d}$ Expression patterns of IMs in three subtypes. e Enrichment disparity of CD274 and PDCD1 across the three subtypes. $\mathbf{f}$ Analysis of stromal and immune scores across the three subtypes: the distribution of samples in C 3 was indicated by the red circle 


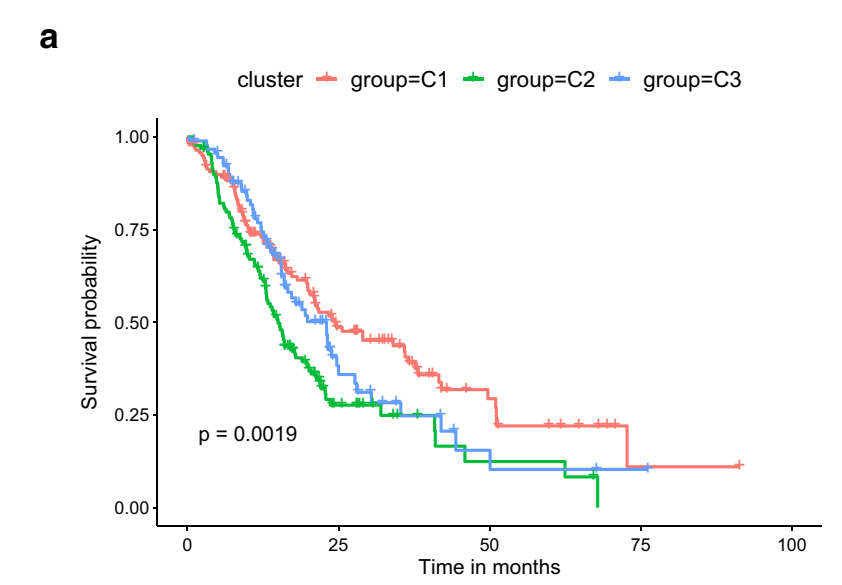

\section{d}

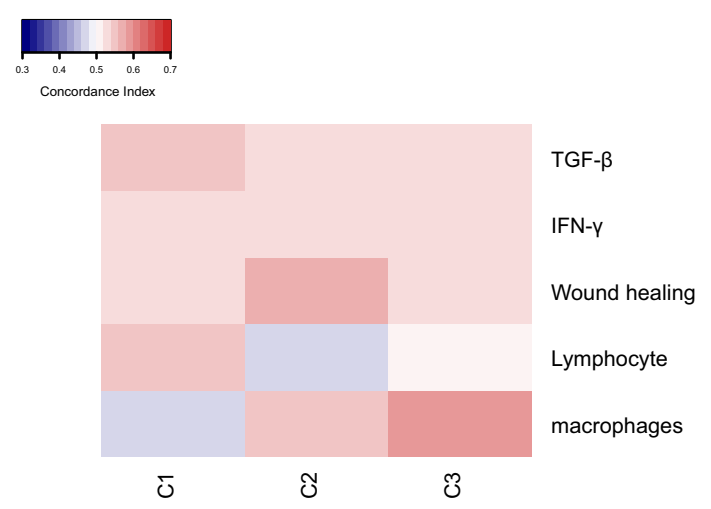

b
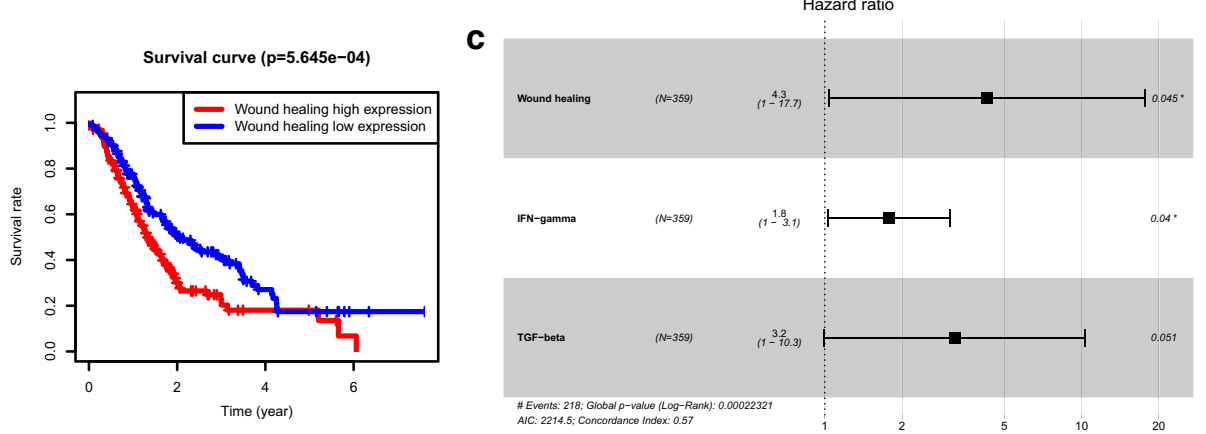

Fig. 4 Prognostic impact of immune subtypes and immune signatures. a Overall survival of three immune subtypes. b Correlation of immune signatures with overall survival. The Left was for IFN- $\gamma$ module. The Right was for Wound healing module. c Multivariate COX analysis of three immune signatures. Wound healing module ( $\mathrm{coef}=1.45, \mathrm{HR}=4.28,95 \% \mathrm{Cl} 1.03-17.67, p=0.045)$, IFN- $\gamma$ module $(\mathrm{coef}=0.57, \mathrm{HR}=1.77,95 \% \mathrm{Cl} 1.03-$ $3.06, p=0.04$ ), TGF- $\beta$ module (coef $=1.16, \mathrm{HR}=3.21,95 \% \mathrm{Cl} 0.99-10.34, p=0.051)$. $\mathbf{d}$ Concordance index (Cl) of five immune signature expression with the overall survival. Columns and rows represented immune subtypes and immune signatures respectively. Red indicated higher prediction accuracy while blue indicated lower prediction accuracy, along with the increasing expression of immune signature enrichment scores

Table 1 Univariate and multivariate cox analyses of five immune signatures

\begin{tabular}{|c|c|c|c|c|c|c|}
\hline \multirow[t]{2}{*}{ Parameter } & \multicolumn{3}{|c|}{ Univariate analysis } & \multicolumn{3}{|c|}{ Multivariate analysis } \\
\hline & HR & $95 \% \mathrm{Cl}$ & $\mathbf{P}$ & HR & $95 \% \mathrm{Cl}$ & $\mathbf{P}$ \\
\hline Wound healing & 9 & $2.38-34.01$ & 0.001 & 4.28 & $1.03-17.67$ & 0.045 \\
\hline Macrophages & 1.29 & $0.67-2.44$ & 0.438 & - & - & - \\
\hline Lymphocyte & 1.06 & $0.60-1.86$ & 0.846 & - & - & - \\
\hline IFN- $\gamma$ & 2.22 & $1.32-3.76$ & 0.003 & 1.77 & $1.03-3.06$ & 0.04 \\
\hline TGF- $\beta$ & 5.42 & $1.80-16.34$ & 0.003 & 3.21 & $0.99-10.34$ & 0.051 \\
\hline
\end{tabular}

C1 and C3 were dominated by low TGM2 expression (Fig. 6c, d). Meanwhile, in the analysis of the immune cells composition between high- and low-TGM2 groups, we found that macrophages mostly enriched in the highTGM2 group, while NKT cells mostly enriched in the low-TGM2 group (Fig. 6e). Furthermore, compared with the low-TGM2 group, the high-TGM2 group seemed to have higher M2 macrophages $(p=0.014)$ and Treg cells $(p=0.068)$ but lower pro-B cells $(p=0.00052)$ and memory B cells ( $p=3.7 \mathrm{e}-6)$ (Fig. 6f, g). And high-TGM2 group harbored a higher expression pattern of inhibitory immunomodulators (CD274, CD276, CTLA4, EDNRB, HAVCR2, LAG3, PDCD1, TGFB1, TIGIT, and VTCN1) in the whole cohort (Fig. 6h). In the prognostic analysis with IMs between the two groups, higher CD276 was associated with worsen prognosis in the high-TGM2 group and higher VTCN1 was related to poorer overall survival in the low-TGM2 group (Fig. 6i). Together, these 


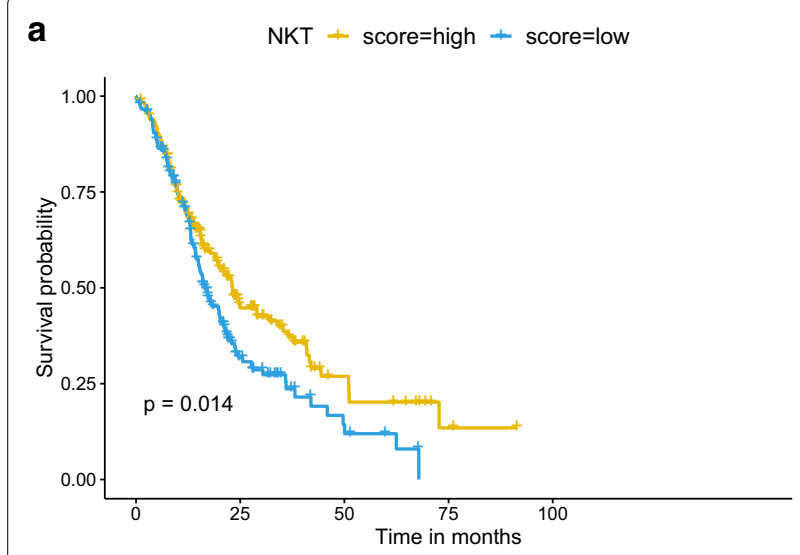

C

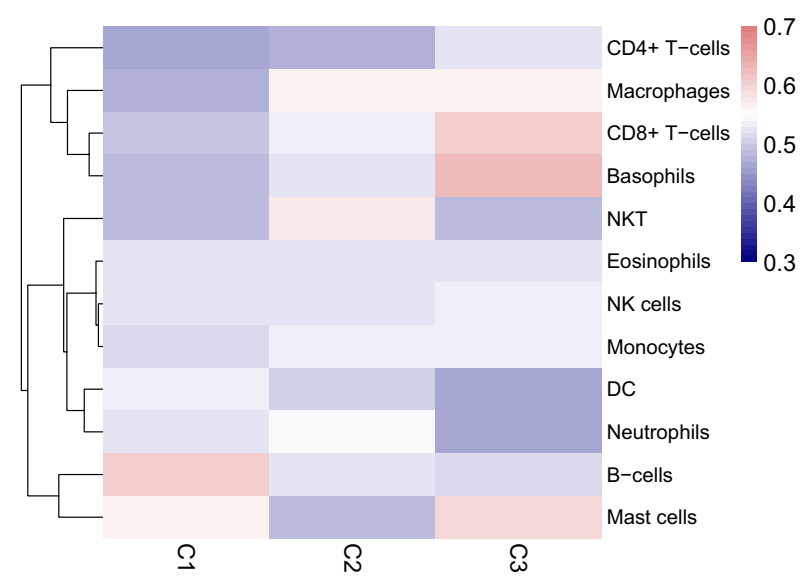

b Macrophages - score=high $\perp$ score=low

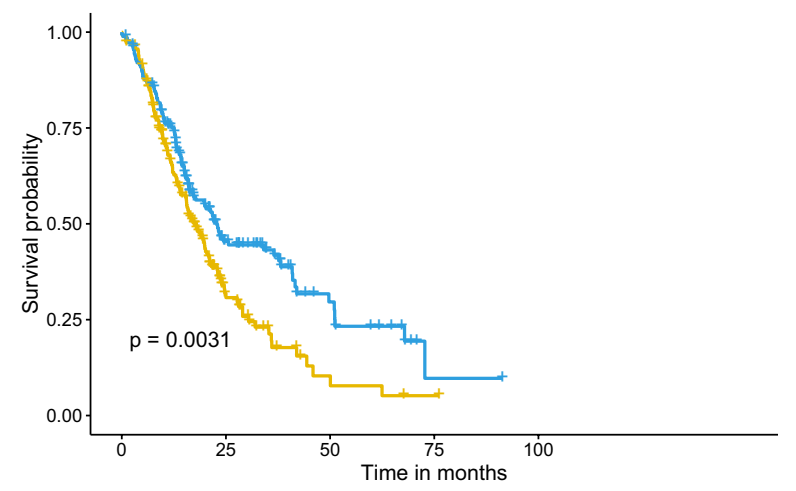

d

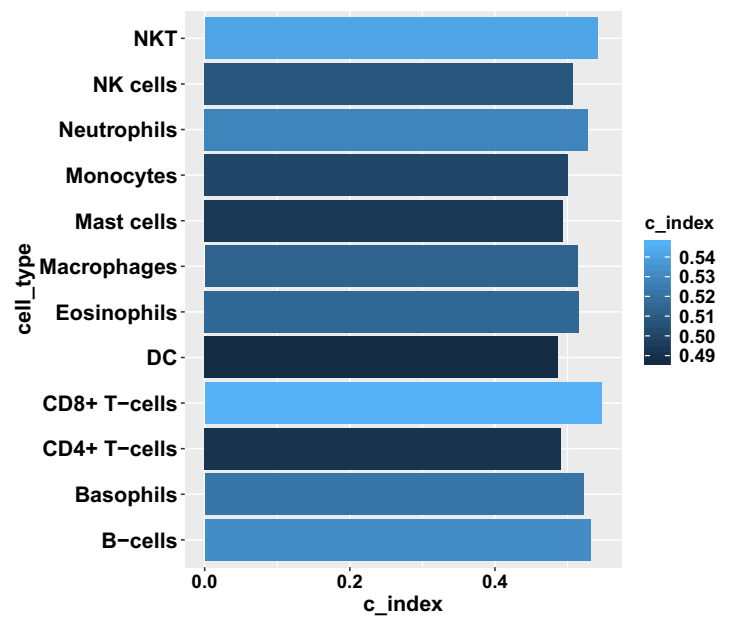

Fig. 5 Prognostic impact of the immune cells. a, b Survival analysis of NKT cells $(p=0.014)$ and macrophages $(p=0.0031)$. $\mathbf{c} \mathrm{Cl}$ analysis with immune cells across the three subtypes: red indicated higher prediction accuracy while blue indicated lower prediction accuracy, along with the increasing expression of immune cells enrichment scores. $\mathbf{d} \mathrm{Cl}$ analysis with immune cells in the whole cohort: the lighter blue indicated greater prognostic impact while the darker blue indicated weaker prognostic impact

findings suggested that TGM2 may be involved in the immunosuppression in PDAC.

\section{TGM2 may regulate PD-L1 expression via STAT3/NF-KB signaling pathways in PDAC}

To further explore the potential roles of TGM2 in immunosuppression, we analyzed the relation between TGM2 and the suppressive factors (Fig. 7a). The relative coefficient between TGM2 and PD-L1 is the most robust (Fig. 7b). We verified the correlation between TGM2 and PD-L1 in human PDAC cell lines (PANC-1 and Mia PaCa-2). After TGM2 knocking-down in PANC-1, we found a decreased expression level of PD-L1 (Fig. 7c). In the cell line of Mia PaCa-2, we observed the same expression variation (Fig. 7d). STAT3 and NF-kB are two important transcriptional factors in tumor evolution and

(See figure on next page.)

Fig. 6 Relation between TGM2 and tumor immunosuppression in PDAC. a IHC analysis of TGM2 with our tissue microarray $(n=97)$. The scale bars were shown as indicated: $100 \mu \mathrm{m}$ and $20 \mu \mathrm{m}$. b Survival analysis of TGM2 with tissue microarray data $(p=0.015)$. c, d Distribution of high- and low-TGM2 group across the three immune subtypes showed as percentage and number. e, $\mathbf{f}$ Immune cell composition of high- and low-TGM2 groups. $\mathbf{g}$ Enrichment comparison of M2 type macrophages $(p=0.014)$, Tregs $(p=0.068)$, pro-B cells $(p=0.00052)$ and memory B cells $(p=3.7 \mathrm{e}-06)$ between high- and low-TGM2 groups. $\mathbf{h}$ Comparison of IMs expression between high- and low-TGM2 groups: row is for the immunomodulators and column is for the gene expression value. (CD274, $p=1.0 \mathrm{e}-14 ; \mathrm{CD} 276, p=1.1 \mathrm{e}-10 ; \mathrm{CTLA4}, p=5.6 \mathrm{e}-07$; CX3CL1, $p=1.6 \mathrm{e}-06$; EDNRB, $p=3.8 \mathrm{e}-06 ; \mathrm{HAVCR} 2, p=4.3 \mathrm{e}-10 ; \mathrm{LAG}, p=0.0046 ; \mathrm{PDCD} 1, p=0.0121 ; \mathrm{TGFB} 1, p=1.2 \mathrm{e}-12 ; \mathrm{TIGIT}, p=6.5 \mathrm{e}-06 ; \mathrm{TLR} 4, p=3.8 \mathrm{e}-08 ; \mathrm{VTCN1}$, $p=0.0585)$. i Survival analysis of CD276 expression in high-TGM2 group (top) and VTCN1 expression in low-TGM2 group (bottom) 
a

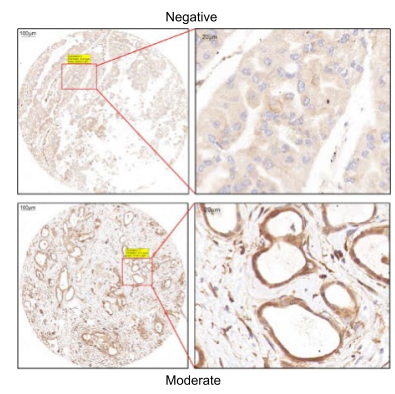

c

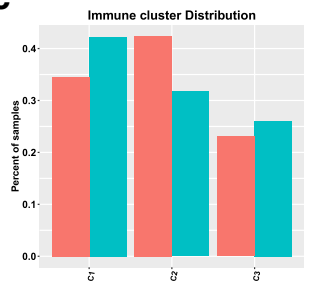

d

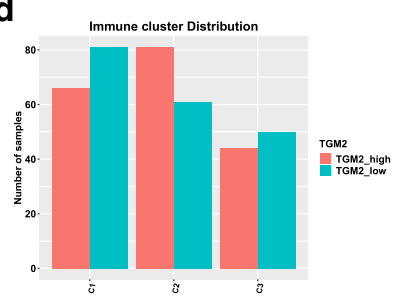

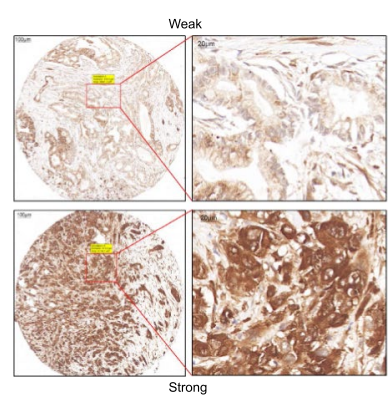

b
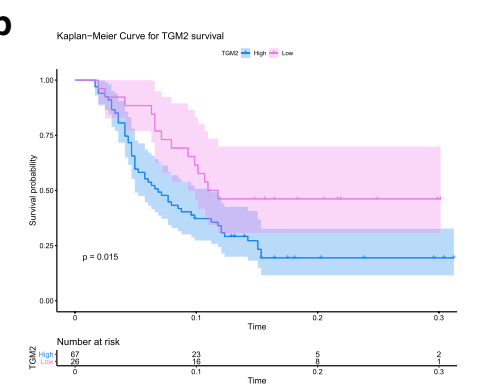

f
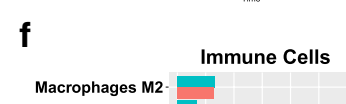

Macrophages M2-

Macrophages M1 -

PDC-

CDC

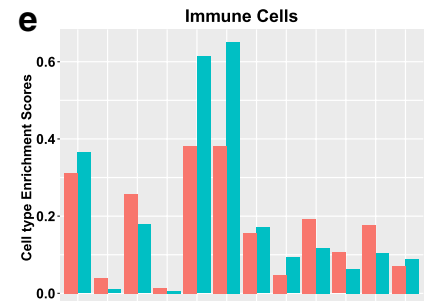

$\mathrm{CDC}$

aDC

CD8+ Tem-

$\mathrm{CD} 8+\mathrm{Tcm}-$

CD8+ naive T-cells -

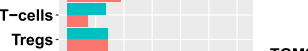

TGM2 TGM2 high
TGM2 low

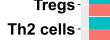

Th2 cells -
Th1 cells -

Tgd cells -

Tgd cells -
CD4+ Tem-

CD4+ Tcm-

CD4+ naive T-cells .

CD4+ memory T-cells -

pro B-cells .

Plasma cells -

naive $\mathrm{B}$-cells -

Memory B-cells -

Memory B-cells -

Cell type Enrichment scores
TGM2

TGM2_high
TGM2_low

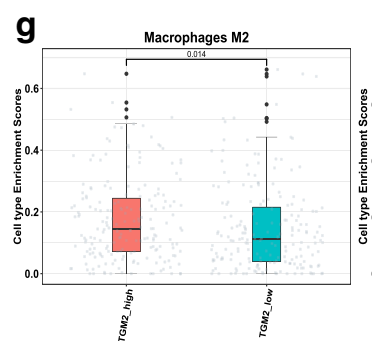

h

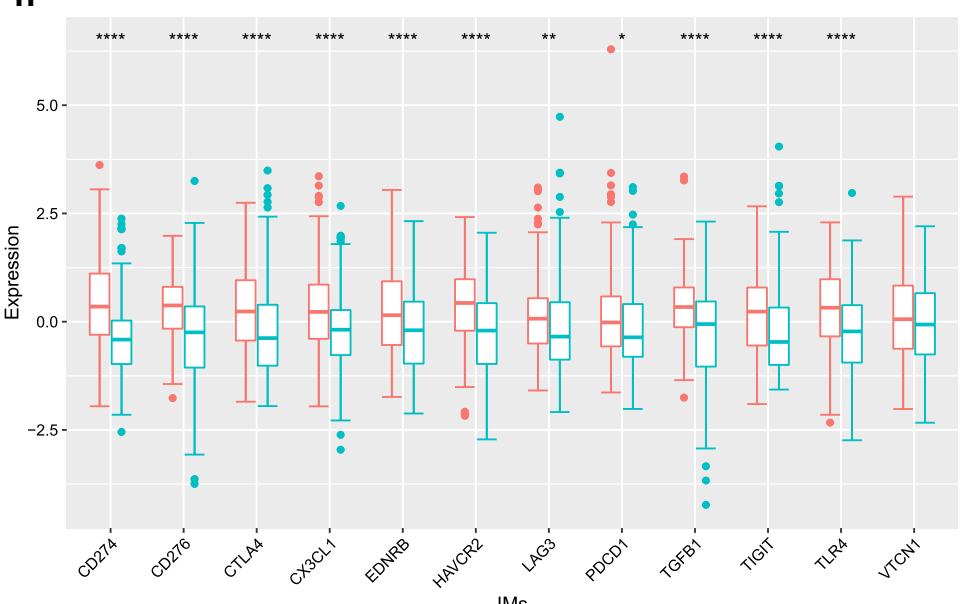

group

官 TGM2_high 户 TGM2_low

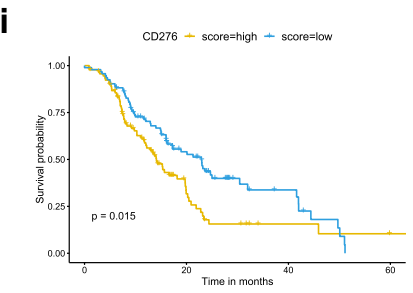

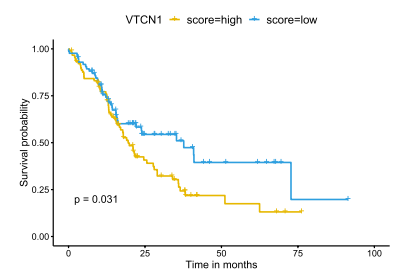



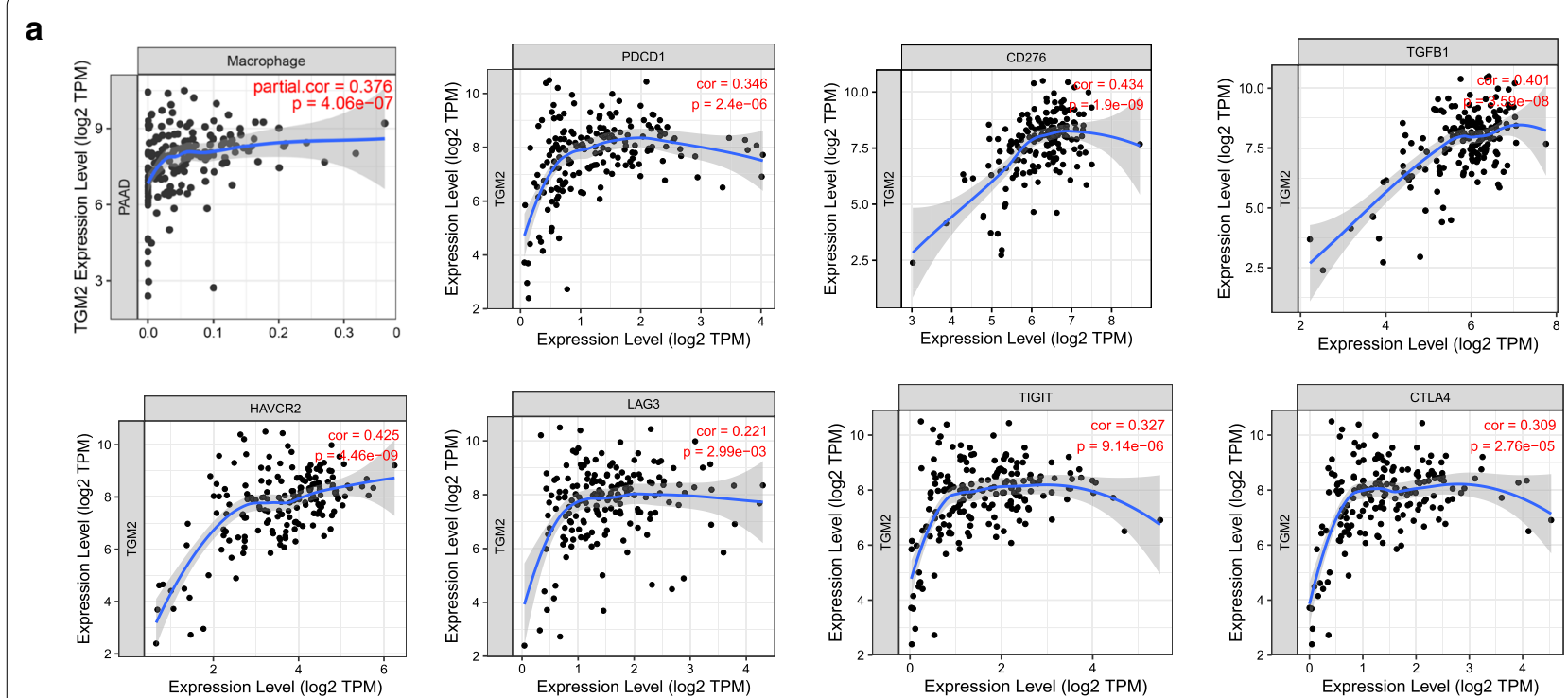

b

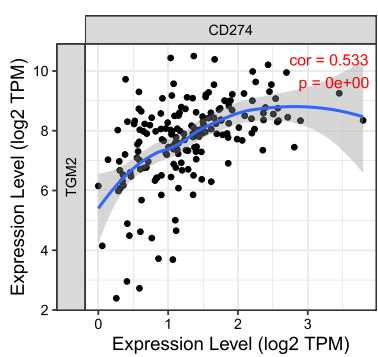

c

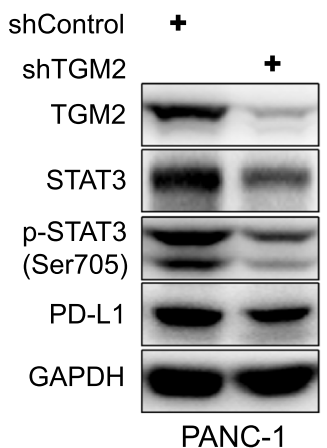

d

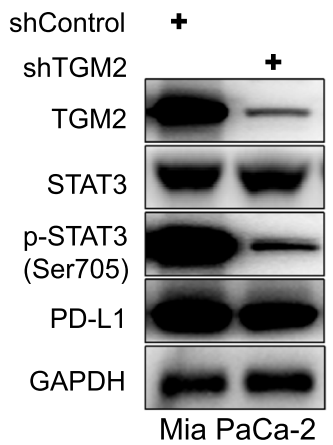

e

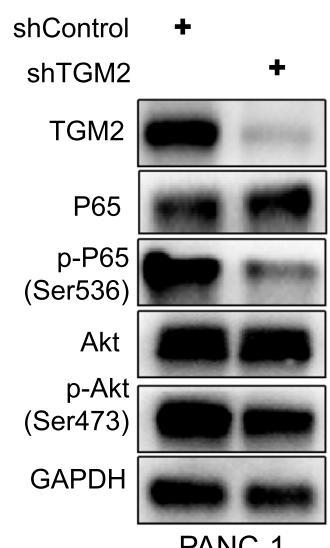

f

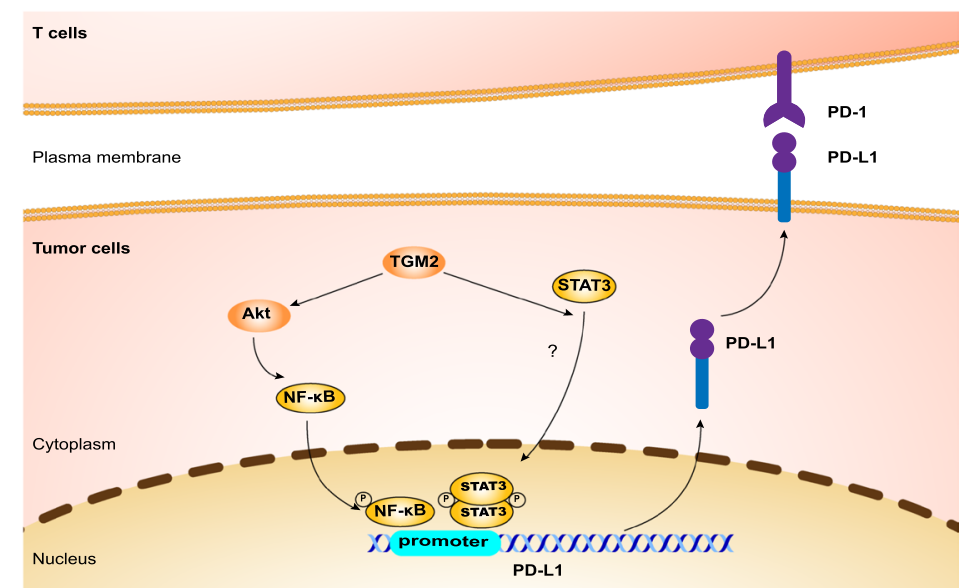

Fig. 7 Proposed mechanism that TGM2 may regulate PD-L1 expression via STAT3/NF-KB signaling pathways in PDAC. a Correlation analysis of TGM2 and immunosuppressive factors with TIMER. $\mathbf{b}$ Correlation analysis of TGM2 and CD274 (PD-L1) by TIMER. $\mathbf{c}$, $\mathbf{d}$ Western blot results showed that TGM2 knocking down resulted in a decreased expression of PD-L1 and p-STAT3 in PANC-1 and Mia PaCa-2 cells. e TGM2 knocking down in PANC-1 cells led to a decreased expression of p-Akt (Ser473) and p-P65 (Ser536) which was consistent with previous studies. f TGM2 may regulate PD-L1 via NF-KB/STAT3 signaling pathways: a TGM2 activated AKT pathway and then promotes the activation of downstream transcription factor NF-KB which has been reported to be able to directly bind with the promoter of PD-L1 and stimulate its transcription. $\mathbf{b}$ TGM2 may promote the phosphorylation of STAT3 despite the underlying pathways remains unclear, and then P-STAT3 binds to the promoter of PD-L1 and stimulate its transcription 
are reported to be capable to regulate the expression of PD-L1 [19, 20]. We knocked down TGM2 in PANC-1 and Mia PaCa-2 cells, a decreasing level of p-STAT3 was observed (Fig. 7c, d). The previous study demonstrated that TGM2 regulated the activation of NF- $\mathrm{kB}$ by modulating phosphorylation of AKT [10]. In our study, we proved that TGM2 knocking down resulted in a decrease of p-Akt (Ser473) and p-P65 (Ser536) (Fig. 7e).

\section{Discussion}

ICB has become a promising immunotherapeutic modality for several refractory carcinomas, however, its roles in PDAC are limited. The challenges majored in the identification of target patients and finding effective combination therapeutic targets to amplify its clinical efficacy. In this study, we utilized published immune signature gene sets to depict the distinct immune features of PDAC and three immune subtypes are identified (the sample details of immune subtypes are shown in the Additional file 1). The prognostic impact of immune gene sets, immune cell composition, and immunomodulators are evaluated. The diversity of immune response reflects the inner cause of the prognostic discrepancy and immunotherapy failure in PDAC. Meanwhile, our study firstly put forward that the high expression of TGM2 tracked with an immunosuppression-promoting phenotype and TGM2 is involved in the modulation of PD-L1 expression by regulating downstream transcription factor STAT3/NF- $\mathrm{kB}$ in pancreatic cancer cells. In addition, the enrichment disparity of IMs in each subtype may suggest multiple potential combination immunotherapy strategies.

The prognostic impact of tumor immune features is emerging to be concerned. Among all subtypes in our study, patients comprising $\mathrm{C} 2$ have the worst overall survival. The reasons for the prognostic discrepancy can be attributed to the following points. Firstly, the immune signatures in C2 are dominated by TGF- $\beta$ and Wound healing modules which were associated with an immunosuppressive and pro-tumor phenotype [21, 22], and inversely correlated with patients prognosis based on our data. Meanwhile, the lowest enrichment degree in Lymphocyte module is another feature of $\mathrm{C} 2$. Consistent with that, the infiltration of T-, B-lymphocytes, and NKT cells in $\mathrm{C} 2$ is less than the other subtypes, indicating a poor preexisting anti-tumor immunity [23]. Moreover, the highest enrichment degree of IFN- $\gamma$ module is found in $\mathrm{C} 2$. IFN- $\gamma$ is a major mediator inducing the death of tumor cells. However, continuous exposure to IFN- $\gamma$ also stimulates tumor cells to express multiple inhibitory modulators including PD-L1 which are majorly enriched in $\mathrm{C} 2$ and $\mathrm{C} 3$ in our study, thereby suppress the secretion of IFN- $\gamma$ by effector $\mathrm{T}$ cells and result in $\mathrm{T}$ cell exhaustion $[24,25]$. Together, a more prominent feature of immunosuppression may be promoted by these features of $\mathrm{C} 2$ and therefore the prognosis of $\mathrm{C} 2$ is worse. In addition to these molecular immune features, our results show that the overall burden of NKT cells is associated with a better prognosis in PDAC, while that is rarely reported before. Similar to $\mathrm{CD} 8^{+} \mathrm{T}$ cell, NKT cell is also regarded as one of the front-line anti-tumor forces [26]. Apart from directly targeting the tumor cells with CD1d positive, recent literature on the mice model demonstrated that NKT cells could restrict the tumor evolution of PDAC indirectly by suppressing the pro-immunosuppression role of macrophages through prostaglandin E synthase-1 (mPGES-1) and 5-lipoxygenase (5-LOX) [27]. Consistent with that, subtype $\mathrm{C} 2$, with a high fraction of macrophage and a low fraction of NKT, shows a more prominent immunosuppression phenotype than $\mathrm{C} 1$ and $\mathrm{C} 3$. These distinct molecular and cellular features across three subtypes show a diverse immune response of PDAC, which would be the condition for prognostic evaluation and immunotherapy strategy designing.

To improve the clinical efficacy of anti-PD1/PDL1 therapy in PDAC, reasonable patient stratification and combination strategy designing should be concerned. For the former, prior studies indicated that the level of preexisting antitumor immunity and the expression level of PD-1/PD-L1 are two vital factors for the efficacy of antiPD-1/PD-L1 treatment [28]. In our study, tumor samples comprising $\mathrm{C} 3$ are rich in various anti-tumor cytokines, such as TNF and IFN- $\gamma$, and have abundant infiltration of immune cells including NKT cells and T lymphocytes as well as B lymphocytes. Despite the anti-tumor effect of B cells remained unclear, a recent study in melanoma indicated that $\mathrm{B}$ cells may contribute to the response to ICB treatment by altering $\mathrm{T}$ cell activation and function [29]. As well, C3 has the highest PD-1 and PD-L1 enrichment across the three subtypes. Thus, patients within the C3 subtype seem to be more suitable for anti-PD treatment. Meanwhile, based on our data, C3 is also rich in CTLA4, TIGIT, and HAVCR2 expression which may provide alternative options for combined targets. Compared with single-agent ICB therapy, combination treatment of antiCTLA4 (ipilimumab) and anti-PDL1 (nivolumab or pembrolizumab) led to better tumor response and patient survival in melanoma, sarcoma, and small cell lung cancer [30-34]. Besides, preclinical studies have demonstrated that anti-TIGIT or anti-HAVCR2 can effectively control tumor evolution, suggesting a promising combination target for anti-PD-1/PD-L1 treatment $[35,36]$.

In contrary with subtype C3, anti-PD-1/PD-L1 treatment may be not appropriate for the patients within subtype $\mathrm{C} 1$ due to the relatively low infiltration of $\mathrm{T} / \mathrm{B}$ lymphocytes and the poorest enrichment in PD-1/ PD-L1. Of note, $\mathrm{C} 1$ has the highest VTCN1 expression 
as well as favorable enrichment scores in NKT cells. As a newly discovered immune checkpoint expressed on APC cells and tumor cells, VTCN1 is expected to become a novel target for immunotherapy in the future despite its regulatory mechanism in cancer immunity remained to be further explored [37]. Due to the advantages of targeted on tumor cells and suppressive effect on graft versus host disease, NKT cell (majorly invariant NKT cell) is regarded as a viable vector for the CAR or rTCR treatments with multiple preclinical animal models supporting favorable anti-tumor effects in solid tumors [38-40].

Subtype C2 has favorable enrichment scores in PD-L1, CD276, and VTCN1 but is poor at lymphocytic infiltration. In addition to anti-PD-L1 treatment to restore the anti-tumor immunity, strategies to target the oncogenic pathway is needed for patients in $\mathrm{C} 2$ to restrict tumor progression. TGM2 is a promising target for improving the response to chemotherapy in solid tumors including PDAC [12]. While its role in the immune evade process of PDAC remains unclear. In this study, we find that TGM2 is positively related to the expression of multiple inhibitory immunomodulators, and the high-TGM2 group is mainly enriched in the immunosuppressive subtype $\mathrm{C} 2$, suggesting that TGM2 may be involved in the regulation of immunosuppression in PDAC. Through in vitro experiments, we verified that TGM2 has a positive impact on PD-L1 in PANC-1 and Mia PaCa- 2 cells. The underlying mechanisms may refer to two aspects. Firstly, STAT3 has been reported to be involved in the regulation of PD-L1 expression as transcriptional factors and confirmed by Chip/EMSA assays [20, 41-43]. However, whether TGM2 alters the expression of PD-L1 in PDAC via STAT3 signaling remains to be unknown. Our present study reveals that down-regulating TGM2 in PANC-1 and Mia PaCa- 2 cells results in a decreased phosphorylation of STAT3, which indicates a potential pathway for the regulation of PD-L1 by TGM2. Secondly, previous studies revealed that TGM2 promotes the activation of AKT by suppressing PTEN, then results in the activation of downstream substances including transcription factor NF-kB in PDAC $[9,10]$. Consistent with that, knocking down TGM2 in PANC-1 cells leads to a decrease in p-Akt and p-P65 expression. As a vital transcriptional factor, NF- $\mathrm{KB}$ can regulate the transcription of PD-L1 by directly binding to the promoter region of PD-L1 [19, 44]. Thus, TGM2 may take a positive impact on PD-L1 expression via Akt/ NF-kB pathway. These findings provide insights into the regulation network of PD-L1 expression in PDAC (Fig. 7f). In addition, inhibition of TGM2 may alleviate stroma fibrosis to facilitate the infiltration of immune effector cells and the entrance of drugs, can also restrain the tumor growth as previous studies reported $[16,45]$. Thereby, targeting on TGM2, through siRNA/shRNA, CRISPR/Cas9 genetic silence, or inhibitor (e.g., miR1285, GK921, NC9) [11, 46-48], may be a promising combination strategy to enhance the sensitivity to anti-PD-1/PD-L1 therapy.

Some limitations in this study need to be addressed. Firstly, as a retrospective study, the clinical value of these findings needs to be further validated in a larger prospective cohort. Secondly, the potential selection bias of tumor specimens may exist. Thirdly, though transcriptional profiles in our study provided us underlying features of the immune response in PDAC, with multiomics data gathering, more solid evidence will promote our insight into tailored treatment for PDAC.

In summary, our study uncovered the specific immune features among PDAC patients. For the discrepant immune response mechanisms, more personalized ICB treatment should be considered. Moreover, TGM2 may regulate the expression of $\mathrm{PD}-\mathrm{L} 1$ in PDAC via STAT3 and Akt/NF-kB signaling pathway and predicts poorer survival of PDAC patients, indicating a potential role in immunotherapy.

\section{Supplementary Information}

The online version contains supplementary material available at https://doi. org/10.1186/s12935-021-01824-z.

Additional file 1: Figure S1. Intergroup prognostic analysis of three immune subtypes. (A) Comparison between C1 and C2 ( $p=0.00094)$. (B) Comparison between C2 and C3 $(p=0.038)$. (C) Comparison between C1 and C3 $(p=0.24)$. Figure S2. Prognostic analysis of B cells, CD8+T cells, NK cells, mast cells, monocytes, DCs, eosinophils, basophils and neutrophils. Figure S3. DEGs and correlation analysis in GEO database (GSE28735 and GSE62452) led to gene TGM2. Figure S4. Prognostic analysis of TGM2 in TCGA-ICGC-GEO cohort ( $p=0.0012$ ). Figure S5. Relation between TGM2 and tumor immunosuppression in PDAC

Additional file 2. The sample details of immune subtypes.

\section{Abbreviations}

AJCC: American Joint Committee on Cancer; GEO: Gene Expression Omnibus; ICB: Immune checkpoint blockade; ICGC: International Cancer Genome Consortium; PDAC: Pancreatic ductal adenocarcinoma; PD-1: Programmed cell death protein 1; PD-L1: Programmed death 1 ligand 1; TGM2: Transglutaminase 2; TCGA: The Cancer Genome Atlas.

\section{Acknowledgments \\ We thank Prof. Yuande Wu and Prof. Xiaolin Yang from the institute of Basic Medical Sciences, Chinese Academy of Medical Sciences.}

\section{Authors' contributions}

$Q L$ and $J L$ designed the research concept of this manuscript. JL wrote the article. QL, XZ and MC reviewed the draft. QL made critical revision. XZ, MC and $T L$ conducted the collection of clinical data and prepared pathological analysis. $J$ ran the bioinformatics analysis of public data. $J L$ and $Y Z$ conducted in vitro experiments. JL checked the data. All authors read and approved the final manuscript.

\section{Funding}

This work was supported by National Natural Science Foundation of China (81673023, 81872501 and 81502068). 


\section{Availability of data and materials}

The public datasets of PAAD, PACA-AU, GSE28735 and GSE62452 for this study can be found in The Cancer Genome Atlas (https://portal.gdc.cancer.gov/), International Cancer Genome Consortium (https://dcc.icgc.org/) and Gene Expression Omnibus (https://www.ncbi.nlm.nih.gov/geo/).

\section{Ethics approval and consent to participate}

The studies involving in human participants were reviewed and approved by the institutional review board of Peking Union Medical Hospital (NO. JS-987).

\section{Consent for publication}

All of the authors agreed to publish this paper in Cancer Cell International.

\section{Competing interests}

The authors declare that they have no competing interests.

Received: 28 October 2020 Accepted: 9 February 2021

Published online: 26 February 2021

\section{References}

1. Siegel RL, Miller KD, Jemal A. Cancer statistics, 2019. CA A Cancer J Clin. 2019:69(1):7-34.

2. Neoptolemos JP, Kleeff J, Michl P, Costello E, Greenhalf W, Palmer DH. Therapeutic developments in pancreatic cancer: current and future perspectives. Nat Rev Gastroenterol Hepatol. 2018;15(6):333-48.

3. Antoni R, Jedd DW. Cancer immunotherapy using checkpoint blockade. Science. 2018;359:1350-5.

4. Macherla S, Laks S, Naqash A, Bulumulle A, Zervos E, Muzaffar M. Emerging role of immune checkpoint blockade in pancreatic cancer. Int J Mol Sci. 2018;19(11):3505.

5. Mahoney KM, Rennert PD, Freeman GJ. Combination cancer immunotherapy and new immunomodulatory targets. Nat Rev Drug Discov. 2015;14(8):561-84.

6. Collisson EA, Bailey P, Chang DK, Biankin AV. Molecular subtypes of pancreatic cancer. Nat Rev Gastroenterol Hepatol. 2019;16(4):207-20.

7. Thorsson V, Gibbs DL, Brown SD, Wolf D, Bortone DS, Ou Yang T-H, PortaPardo E, Gao GF, Plaisier CL, Eddy JA, et al. The immune landscape of cancer. Immunity. 2018;48(4):812-830.e814.

8. Fesus L, Piacentini M. Transglutaminase 2: an enigmatic enzyme with diverse functions. Trends Biochem Sci. 2002;27(10):534-9.

9. Mann AP, Verma A, Sethi G, Manavathi B, Wang H, Fok JY, Kunnumakkara $A B$, Kumar R, Aggarwal BB, Mehta K. Overexpression of tissue transglutaminase leads to constitutive activation of nuclear factor-kB in cancer cells: delineation of a novel pathway. Can Res. 2006;66(17):8788-95.

10. Verma A, Guha S, Wang H, Fok JY, Koul D, Abbruzzese J, Mehta K. Tissue transglutaminase regulates focal adhesion kinase/AKT activation by modulating PTEN expression in pancreatic cancer cells. Clin Cancer Res. 2008;14(7):1997-2005.

11. Lee S-H, Lee W-K, Kim N, Kang J, Kim K-H, Kim S-G, Lee J-S, Lee S, Lee J, Joo J, et al. Renal cell carcinoma is abrogated by p53 stabilization through transglutaminase 2 inhibition. Cancers. 2018;10(11):455.

12. Eckert RL, Fisher ML, Grun D, Adhikary G, Xu W, Kerr C. Transglutaminase is a tumor cell and cancer stem cell survival factor. Mol Carcinog. 2015;54(10):947-58.

13. Zhang H, Chen Z, Miranda RN, Medeiros LJ, McCarty N. TG2 and NF-KB signaling coordinates the survival of mantle cell lymphoma cells via IL6mediated autophagy. Can Res. 2016;76(21):6410-23.

14. Mehta K, Han A. Tissue transglutaminase (TG2)-induced inflammation in initiation, progression, and pathogenesis of pancreatic cancer. Cancers. 2011;3(1):897-912.

15. Verma A, Guha S, Diagaradjane P, Kunnumakkara AB, Sanguino AM, Lopez-Berestein G, Sood AK, Aggarwal BB, Krishnan S, Gelovani JG, et al. Therapeutic significance of elevated tissue transglutaminase expression in pancreatic cancer. Clin Cancer Res. 2008:14(8):2476-83.

16. Lee J, Condello S, Yakubov B, Emerson R, Caperell-Grant A, Hitomi $\mathrm{K}$, Xie J, Matei D. Tissue transglutaminase mediated tumor-stroma interaction promotes pancreatic cancer progression. Clin Cancer Res. 2015:21(19):4482-93.
17. Liu Q, Wu H, Li Y, Zhang R, Kleeff J, Zhang X, Cui M, Liu J, Li T, Gao J, et al. Combined blockade of TGf- $\beta 1$ and GM-CSF improves chemotherapeutic effects for pancreatic cancer by modulating tumor microenvironment. Cancer Immunol Immunother. 2020;69(8):1477-92.

18. Amin MB, Greene FL, Edge SB, Compton CC, Gershenwald JE, Brookland RK, Meyer L, Gress DM, Byrd DR, Winchester DP. The eighth edition AJCC cancer staging manual: continuing to build a bridge from a populationbased to a more "personalized" approach to cancer staging. CA A Cancer J Clin. 2017:67(2):93-9.

19. Wang W, Chapman NM, Zhang B, Li M, Fan M, Laribee RN, Zaidi MR, Pfeffer LM, Chi H, Wu Z-H. Upregulation of PD-L1 via HMGB1-activated IRF3 and NF-kB contributes to UV radiation-induced immune suppression. Can Res. 2019;79(11):2909-22.

20. Wang B, Zhou Y, Zhang J, Jin X, Wu H, Huang H. Fructose-1,6-bisphosphatase loss modulates STAT3-dependent expression of PD-L1 and cancer immunity. Theranostics. 2020;10(3):1033-45.

21. Haibe-Kains B, Wolf DM, Lenburg ME, Yau C, Boudreau A, van't Veer LJ. Gene co-expression modules as clinically relevant hallmarks of breast cancer diversity. PLoS ONE. 2014;9(2):e88309.

22. Edison TL, Chang HY, Sneddon JB, Alizadeh AA, Sood R, West RB, Montgomery K, Chi JT, Rijn MVD, Botstein D, et al. Gene expression signature of fibroblast serum response predicts human cancer progression: similarities between tumors and wounds. PLoS Biol. 2004;2(2):e7.

23. Calabrò A, Beissbarth T, Kuner R, Stojanov M, Benner A, Asslaber M, Ploner F, Zatloukal K, Samonigg H, Poustka A, et al. Effects of infiltrating lymphocytes and estrogen receptor on gene expression and prognosis in breast cancer. Breast Cancer Res Treat. 2008;116(1):69-77.

24. Zerdes I, Matikas A, Bergh J, Rassidakis GZ, Foukakis T. Genetic, transcriptional and post-translational regulation of the programmed death protein ligand 1 in cancer: biology and clinical correlations. Oncogene. 2018:37(34):4639-61.

25. Saka D, Gökalp M, Piyade B, Cevik NC, Arik Sever E, Unutmaz D, Ceyhan $\mathrm{GO}$, Demir IE, Asimgil H. Mechanisms of T-cell exhaustion in pancreatic cancer. Cancers. 2020;12(8):2274.

26. Terabe M, Berzofsky JA. Tissue-specific roles of NKT cells in tumor immunity. Front Immunol. 1838;2018:9.

27. Janakiram NB, Mohammed A, Bryant T, Ritchie R, Stratton N, Jackson L, Lightfoot S, Benbrook DM, Asch AS, Lang ML, et al. Loss of natural killer T cells promotes pancreatic cancer in LSL-KrasG12D/+ mice. Immunology. 2017;152(1):36-51.

28. Sanmamed MF, Chen L. A paradigm shift in cancer immunotherapy: from enhancement to normalization. Cell. 2018:175(2):313-26.

29. Helmink BA, Reddy SM, Gao J, Zhang S, Basar R, Thakur R, Yizhak K, SadeFeldman M, Blando J, Han G, et al. B cells and tertiary lymphoid structures promote immunotherapy response. Nature. 2020;577(7791):549-55.

30. Rotte A. Combination of CTLA-4 and PD- 1 blockers for treatment of cancer. J Exp Clin Cancer Res. 2019;38(1):1-12.

31. Larkin J, Chiarion-Sileni V, Gonzalez R, Grob JJ, Cowey CL, Lao CD, Schadendorf D, Dummer R, Smylie M, Rutkowski P, et al. Combined nivolumab and ipilimumab or monotherapy in untreated melanoma. N Engl J Med. 2015;373(1):23-34.

32. Long GV, Atkinson V, Cebon JS, Jameson MB, Fitzharris BM, McNeil CM, Hill AG, Ribas A, Atkins MB, Thompson JA, et al. Standard-dose pembrolizumab in combination with reduced-dose ipilimumab for patients with advanced melanoma (KEYNOTE-029): an open-label, phase $1 \mathrm{~b}$ trial. Lancet Oncol. 2017;18(9):1202-10.

33. D'Angelo SP, Mahoney MR, Van Tine BA, Atkins J, Milhem MM, Jahagirdar BN, Antonescu CR, Horvath E, Tap WD, Schwartz GK, et al. Nivolumab with or without ipilimumab treatment for metastatic sarcoma (Alliance A091401): two open-label, non-comparative, randomised, phase 2 trials. Lancet Oncol. 2018;19(3):416-26.

34. Antonia SJ, López-Martin JA, Bendell J, Ott PA, Taylor M, Eder JP, Jäger D, Pietanza MC, Le DT, de Braud F, et al. Nivolumab alone and nivolumab plus ipilimumab in recurrent small-cell lung cancer (CheckMate 032): a multicentre, open-label, phase 1/2 trial. Lancet Oncol. 2016;17(7):883-95.

35. Harjunpää H, Guillerey C. TIGIT as an emerging immune checkpoint. Clin Exp Immunol. 2019;200(2):108-19.

36. Sakuishi K, Apetoh L, Sullivan JM, Blazar BR, Kuchroo VK, Anderson AC. Targeting Tim-3 and PD-1 pathways to reverse T cell exhaustion and restore anti-tumor immunity. J Exp Med. 2010;207(10):2187-94. 
37. Podojil JR, Miller SD. Potential targeting of B7-H4 for the treatment of cancer. Immunol Rev. 2017;276(1):40-51.

38. Wolf BJ, Choi JE, Exley MA. Novel approaches to exploiting invariant NKT cells in cancer immunotherapy. Front Immunol. 2018;9:384.

39. Heczey A, Liu D, Tian G, Courtney AN, Wei J, Marinova E, Gao X, Guo L, Yvon E, Hicks J, et al. Invariant NKT cells with chimeric antigen receptor provide a novel platform for safe and effective cancer immunotherapy. Blood. 2014;124(18):2824-33.

40. Tian G, Courtney AN, Jena B, Heczey A, Liu D, Marinova E, Guo L, Xu X Torikai H, Mo Q, et al. CD62 L+ NKT cells have prolonged persistence and antitumor activity in vivo. J Clin Invest. 2016;126(6):2341-55.

41. Marzec M, Zhang Q, Goradia A, Raghunath PN, Liu X, Paessler M, Wang HY, Wysocka M, Cheng M, Ruggeri BA, et al. Oncogenic kinase NPM/ALK induces through STAT3 expression of immunosuppressive protein CD274 (PD-L1, B7-H1). Proc Natl Acad Sci USA. 2008;105:20852-7.

42. Zerdes I, Wallerius M, Sifakis EG, Wallmann T, Betts S, Bartish M, Tsesmetzis N, Tobin NP, Coucoravas C, Bergh J, et al. STAT3 activity promotes programmed-death ligand 1 expression and suppresses immune responses in breast cancer. Cancers. 2019;11(10):1479.

43. Bu LL, Yu GT, Wu L, Mao L, Deng WW, Liu JF, Kulkarni AB, Zhang WF, Zhang $L$, Sun ZJ. STAT3 induces immunosuppression by upregulating PD-1/ PD-L1 in HNSCC. J Dent Res. 2017;96(9):1027-34.

44. Cheriyath V, Gowrishankar K, Gunatilake D, Gallagher SJ, Tiffen J, Rizos $H$, Hersey P. Inducible but not constitutive expression of PD-L1 in human melanoma cells is dependent on activation of NF-KB. PLOS ONE. 2015;10(4):e0123410

45. Verma A, Wang H, Manavathi B, Fok JY, Mann AP, Kumar R, Mehta K. Increased expression of tissue transglutaminase in pancreatic ductal adenocarcinoma and its implications in drug resistance and metastasis. Can Res. 2006;66(21):10525-33.

46. Hidaka H, Seki N, Yoshino H, Yamasaki T, Yamada Y, Nohata N, Fuse M, Nakagawa M, Enokida H. Tumor suppressive microRNA-1285 regulates novel molecular targets: aberrant expression and functional significance in renal cell carcinoma. Oncotarget. 2012;3(1):44-57.

47. Ku BM, Kim SJ, Kim N, Hong D, Choi YB, Lee SH, Gong YD, Kim SY. Transglutaminase 2 inhibitor abrogates renal cell carcinoma in xenograft models. J Cancer Res Clin Oncol. 2014;140(5):757-67.

48. Adhikary G, Grun D, Alexander HR, Friedberg JS, Xu W, Keillor JW, Kandasamy S, Eckert RL. Transglutaminase is a mesothelioma cancer stem cell survival protein that is required for tumor formation. Oncotarget. 2018;9(77):34495-505.

\section{Publisher's Note}

Springer Nature remains neutral with regard to jurisdictional claims in published maps and institutional affiliations.
Ready to submit your research? Choose BMC and benefit from:

- fast, convenient online submission

- thorough peer review by experienced researchers in your field

- rapid publication on acceptance

- support for research data, including large and complex data types

- gold Open Access which fosters wider collaboration and increased citations

- maximum visibility for your research: over 100M website views per year

At BMC, research is always in progress.

Learn more biomedcentral.com/submissions 